\title{
Quantitative risk assessment of acquiring listeriosis from consumption of minimally processed vegetables.
}

\begin{abstract}
Listeriosis is a serious invasive disease with high fatality rates especially among the vulnerable population. Although the causative pathogen had been detected in several foods in Malaysia, there have so far been no reported cases of listeriosis in Malaysia. The aim of this study was to carry out a quantitative risk assessment to estimate the risk of acquiring listeriosis through the consumption of minimally processed salad vegetables in Malaysia. The probability of illness per serving was calculated using an exponential dose-response model for high-risk and low-risk groups of consumer using the stochastic approach. The risk estimate of acquiring listeriosis for the healthy population was $1.98 \times 10-4$ per 100,000 population. For susceptible population, the risk estimate was considerably higher, i.e., 0.172 per 100,000 population for AIDS patients, 0.00496 per 100,000 population for diabetics and 0.149 per 100,000 for the elderly population.
\end{abstract}

Keyword: Listeriosis; Vegetables; Minimally pr 